\title{
ADA in San Francisco, 13-17 June 2014
}

\section{Dr Caroline Day reports from the 74th Annual Scientific Sessions of the American Diabetes Association}

\section{Introduction}

At the 74th Scientific Sessions of the ADA more than 16,000 delegates from around the world converged on the Moscone Centre in downtown San Francisco to be presented with a palette of sessions running from 8am to 6.30pm - plus pre-breakfast and pre-supper CME events - sightseeing and sleep were not high on the agenda. For most of the meeting there were 8 parallel sessions which included 90 symposia, 50 oral sessions presenting 383 abstracts, plus poster sessions where more than 1,700 posters were displayed in addition to the 307 "late breakers". The 10 professional interest groups held their discussions over lunch and large TV screens drew specialist audiences at critical points in the FIFA World Cup qualifying series.

\section{Named lectures}

There were 10 special lectures and addresses, including the Roger Pecardo Award Lecture delivered by Professor William Jeffcoate (Nottingham, UK) who highlighted the need for multidisciplinary care to combat the chronic nature of the diabetic foot. He suggested that a greater understanding of wound healing mechanisms in diabetes is needed to develop more effective treatments.

Daniel J Drucker (Toronto, Canada), this year's recipient of the Banting Medal for Scientific Achievement, delivered a well crafted, informative and entertaining lecture. He noted that it was only in the early 1980s that the gut emerged as a major endocrine organ. Several GLPs have been discovered and the therapeutic potential of GLP-1 quickly became apparent. However it has taken another 30 years for these findings to deliver the DPP-4 inhibitors and GLP1 receptor agonists (and further modalities in pharmaceutical development). The nonglycaemic actions of GLP-1 offer wide ranging therapeutic potential from an anti-obesity effect to neuroprotection. Indeed a GLP-2 receptor agonist (teduglutide), which increases intestinal absorptive capacity, has reduced or negated dependence on parenteral nutrition in patients with short bowel syndrome. Dr Drucker concluded "The glucagon gene has already produced more approved drugs than any other gene in the human body; and there remains much more work to be done".

\section{Prevention}

The positive outcomes of the US Diabetes Prevention Program (1996-1999; reduced risk of progression to type 2 diabetes: $58 \%$ with intensive lifestyle, 31\% with metformin; versus placebo) led to its continuation as an outcome study - DPPOS. In $2013,87 \%$ of the original cohort were still in the study, differences in prevalence between treatment strategies continued: reduction in type 2 diabetes ( $27 \%$ and $18 \%$ ); reduced duration of diabetes (1.1 and 0.6 years); lower body weight (3-4\% and 1$2 \%$ ) in the original intensive lifestyle and metformin treated groups respectively compared with placebo. Mean $\mathrm{HbA}_{1 \mathrm{c}}(0.1 \%)$ was also lower.

Subjects on metformin who had an initial $\geq 5 \%$ drop in body weight maintained a weight reduction $(\sim 2 \mathrm{~kg})$ over the 15 years of the study (better than most anti-obesity agents). In 10,000 subject years of follow-up metformin-associated vitamin B12 malabsorption was not an issue $(12 \%$ decrease in haematocrit versus $8.4 \%$ in placebo), but vitamin B12 deficient subjects on metformin experienced more severe neuropathy. Intensive lifestyle advice $(\$ 10,760$ per QALY gained compared with placebo) and metformin were considered cost effective interventions.

Translation of the lifestyle intervention curriculum into a year-long programme delivered in senior community centres was effective in improving weight, physical activity and risk factors for metabolic syndrome. Analysis of the VA MOVE programme (a 'real world' diet and exercise initiative for veterans) produced similar benefits.

\section{Treatment}

The merits of the latest ACC-AHA lipid guideline were debated. A pertinent point was that the guideline was meant to inform, not to mandate. There was consensus that lifestyle recommendations were similarly relevant to those with and without diabetes and that lack of a recommended target for LDL was unhelpful, as was recommending high dose statin use in most patients. It was noted that the mild adverse events commonly associated

\begin{tabular}{|c|c|}
\hline ACC-AHA & $\begin{array}{l}\text { American College of Cardiology - } \\
\text { American Heart Association }\end{array}$ \\
\hline ADA & American Diabetes Association \\
\hline CME & continuing medical education \\
\hline CV & cardiovascular \\
\hline DPP-4 & dipeptidylpeptidase-4 \\
\hline DPPOS & $\begin{array}{l}\text { Diabetes Prevention Program } \\
\text { Outcome Study }\end{array}$ \\
\hline FIFA & $\begin{array}{l}\text { Fédération Internationale de } \\
\text { Football Association }\end{array}$ \\
\hline GLP & glucagon-like peptide \\
\hline $\mathrm{HbA}_{1 \mathrm{c}}$ & glycated haemoglobin $1 \mathrm{c}$ \\
\hline LDL & low density lipoprotein \\
\hline QALY & quality-adjusted life years \\
\hline RCT & randomised controlled trial \\
\hline SGLT2 & sodium glucose co-transporter 2 \\
\hline
\end{tabular}

with statin use could usually be remedied by a change of statin. There was concern that only using RCT data to derive guidance ignored decades of knowledge. Nevertheless the benefit:risk decision came out in favour of statin use, with the proviso that treatment strategies are individualised.

The ongoing conundrums of treating diabetes were addressed, unravelling the relationship between hypoglycaemia and CV disease, the CV benefit of glycaemic control, the implications of CV outcomes in recent diabetes trials, the value of screening for subclinical CV disease, investigating and treating complications as well as educational and behavioural approaches. The benefits of exercise (in all its forms - including standing) were extolled, but it was highlighted that about $7 \%$ of people do not experience the extent of improvements enjoyed by the majority. Discussion of the revised ADA nutrition guidance clarified it as the established mantra re-packaged in a patient-friendly format: 'healthy eating patterns'.

Studies on current glucose lowering drugs abounded and much interest was generated by the four experts (including the UK's Professor Guy Rutter) who explored the effects of the GLP-1 receptor agonists on the exocrine and endocrine pancreas. A session on the newest class of agents, the SGLT2 inhibitors, drew an overflow audience (>4000), and included a review by BJDVD's editor Professor Cliff Bailey. Several sessions focussed on the microbiome 
and showed how its composition and activity altered with diet, how the maternal microbiome varies during lactation, differs between normal weight and obese mothers and how the maternal microbiome is passed to the baby. Despite many differences being noted, causal relationships with diabetes and $\mathrm{CV}$ risk were not identified.

The ADA always has an eye to the longterm future of diabetes, and sessions focussed on closed-loop insulin delivery and pancreas and islet transplantation generated considerable discussion. Newcastle's Professor Jim Shaw assessed recent advances in islet transplantation and described substantial challenges preventing its more widespread use.

\section{The personal touch}

Several studies investigated patient outcomes in relation to the professional qualifications of the diabetes specialist delivering their care. No significant differences were noted between nurse practitioners, physician's assistants, pharmacists and physicians. Of interest was a trial showing the benefits of addition of healthcare coaches to urban 'safety net' clinics. After 12 months nearly half of the patients had improved at least 1 of 3 CV risk factors and more than twice as many patients receiving coaching had achieved their $\mathrm{HbA}_{1 c}$ targets.

\section{Finally....}

ADA 2015 will be in Boston, Massachusetts. In the interim, avoid weight gain, consume a balanced diet and take regular exercise.

Dr Caroline Day Email cday@mededuk.com

http://dx.doi.org/10.15277/bjdvd.2014.034 $\mathrm{Br}$ J Diabetes Vasc Dis 2014;14:117-18

\section{CARDIORENAL FORUM}

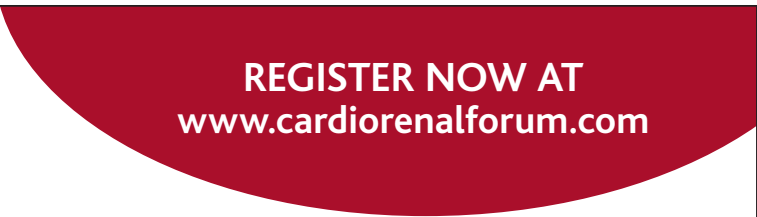

\section{9th Annual Scientific Meeting of the Cardiorenal Forum}

\section{Cardiorenal disease: glucose, gender, rhythm and risks}

Friday 3rd October 20149.30 am to 5.15 pm

RCOG, Regent's Park, London, NW1 4RG

Endorsed by the Renal Association and British Society for Heart Failure 6 CPD credits approved by the Royal College of Physicians Registration fee only $£ 75$ (or $£ 35$ concessions)

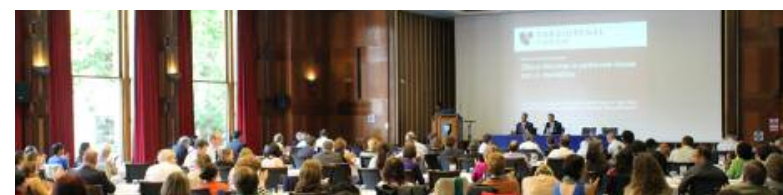

Session 1. Diabetes and cardiorenal disease

- Professor Kay Tee Khaw (University of Cambridge)

- Professor Cliff Bailey (Aston University, Birmingham)

- Dr Phil McEwan (Swansea Centre for Health Economics)

- Dr John Holian (St Vincent's University Hospital, Dublin)

Session 2. Advances in diagnosis and assessing CV risk

- Professor John Deanfield (University College Hospital, London)

- Dr Anthony Wierzbicki (Guy's \& St Thomas' Hospital, London)

- Dr Mike Polkey (Royal Brompton Hospital, London)

- Professor David Goldsmith (Guy's and St Thomas' Hospital, London)

- $\quad$ Professor lain Squire (University of Leicester)

- Professor David Wheeler (Royal Free Hospital, London)

Session 3. New considerations in cardiorenal risk

- Professor Peter Collins (Imperial College, London)

- Professor Adelbert Schiller (Timisoara, Romania)

Session 4. Interactive cases

- $\quad$ Dr Diana Gorog (East \& North Hertfordshire NHS Trust)

- Dr Daniel Sado (University College Hospital, London)

- Professor Andrew Clark (University of Hull) 